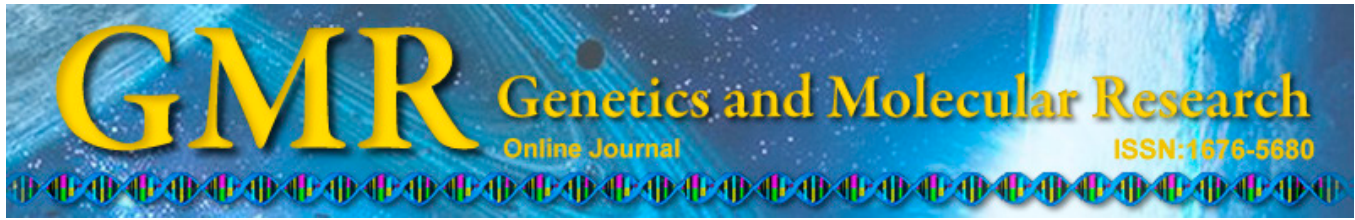

\title{
First transcriptional survey of the Malpighian tubules of giant mealworm, Zophobas morio (Coleoptera: Tenebrionidae)
}

\author{
J.R. Silva ${ }^{1,2}$, R.A. Prado ${ }^{1}$, D.T. Amaral ${ }^{1,2}$ and V.R. Viviani ${ }^{1,2}$ \\ ${ }^{1}$ Programa de Pós-Graduação em Biotecnologia e Monitoramento Ambiental, \\ Universidade Federal de São Carlos, Sorocaba, SP, Brasil \\ ${ }^{2}$ Programa de Pós-Graduação em Genética Evolutiva e Biologia Molecular, \\ Universidade Federal de São Carlos, São Carlos, SP, Brasil \\ Corresponding author: V.R. Viviani \\ E-mail: viviani@ufscar.br
}

Genet. Mol. Res. 14 (1): 464-473 (2015)

Received May 12, 2014

Accepted October 23, 2014

Published January 23, 2015

DOI http://dx.doi.org/10.4238/2015.January.23.21

\begin{abstract}
The Malpighian tubules play a key role in insect osmoregulation. Although a transcriptional analysis has been done for the Malpighian tubules in Drosophila melanogaster (Diptera), no functional genomics analysis has yet been carried out for any Coleoptera species. Recently, we constructed a cDNA library from Malpighian tubules of larval Zophobas morio, a close relative of Tribolium castaneum, and cloned the cDNA for an AMP/CoA-ligase with luciferase-like enzyme properties. Using this cDNA library, we randomly isolated, partially sequenced and analyzed ca. 540 clones, obtaining the first transcriptional profile of the most representative expressed genes, and associated them with their possible biological functions. A high percentage of mitochondrial genes was found, which is consistent with the high metabolic activity required by this organ during the formation of primary urine. Common transcripts included those for enzymes involved in osmoregulation, such as solute transporters and ATPases, and in detoxification and excretion, such as cytochrome
\end{abstract}


P450, glutathione S-transferase, alcohol dehydrogenase. The presence of AMP/CoA-ligases, which activate exogenous carboxylic acids such as firefly D-luciferin suggests their participation in important new xenobiotic excretion/detoxification roles in Malpighian tubule physiology.

Key words: AMP/CoA-ligase; Excretion; Detoxification; Transcriptome

\section{INTRODUCTION}

The Malpighian tubules in insects play an essential role in osmoregulation, performing transport of ions and fluids, during the formation of primary urine (Wigglesworth, 1972). They carry out active excretion of a wide variety of toxic compounds such as nicotine (Gaertner et al., 1998), ouabain (Torrie et al., 2004), salicylate (Ruiz-Sanchez et al., 2007), cardiac glycosides (Rafaeli-Bernstein and Mordue, 1978), morphine, and atropine (Maddrell and Gardiner, 1976). Studies have shown that Drosophila melanogaster Malpighian tubules are resistant to ouabain (ATPase $\mathrm{Na}^{+}, \mathrm{K}^{+}$inhibitor) due to the dominant presence of carrier proteins that have a broad spectrum of substrates (Torrie et al., 2004). The ability to eliminate toxic compounds is important for insects which feed on alkaloid-containing plants, and it also allows the insects to explore a broad range of food plants, which is important for adaptation of insects to a wide range of habitats and diets (Maddrell and Gardiner, 1976, Klowden, 2007).

Besides the general function of excretion and formation of urine, the Malpighian tubules may also be involved in other specialized functions in insects, such as immunologic response through the secretion of antimicrobial peptides (Nappi et al., 2000), production of silk in modified tubules of Diptera and Neuroptera larvae (Wigglesworth, 1972), storage of calcium in the Malpighian tubules of Musca autumnalis during hardening of the pupae (Krueger et al., 1988), and production of bioluminescence from the modified terminal ends of Malpighian tubules of Arachnocampa spp larvae for prey attraction purposes (Gatenby, 1960; Viviani et al., 2002). The Malpighian tubules of insects are also good models for human kidney diseases, displaying similar genes as those involved in human genetic diseases (Dow and Davies, 2006).

A functional genomic analysis of the Malpighian tubules in D. melanogaster suggested more extensive functions than just ion and water transport, where they could be involved with the rectum in the excretion of a broad range of organic solutes and xenobiotics (Gaertner et al., 1998; Dow and Davies, 2006). High levels of cytochrome P450, alcohol dehydrogenases and gluthatione S-transferases were found to be expressed in D. melanogaster Malpighian tubules, these enzymes participate in the metabolism of potentially toxic compounds (Dow and Davies, 2006; Yang et al., 2007). The Malpighian tubules also display an abundance of transcription factors, which may be involved in the regulation of specific functions in this tissue (Wang et al., 2004).

Despite the considerable interest in Malpighian tubules in the physiology of beetles, no transcriptional profile has yet been determined for any Coleoptera. Considering that the genomes of various insects (D. melanogaster, Anopheles gambiae, Apis mellifera, and Tribolium castaneum) have been sequenced, there is a rich database for analysis of expressed genes and inference of their functions.

The tenebrionid dark-beetles such as Tenebrio molitor and Zophobas morio are spe- 
cies closely related to $T$. castaneum, where the larvae are commonly known as giant mealworms, which are commercially available in pet shops as a food source and as a lure for fishing purposes. These larvae are easily reared on flour, providing a ready source of biological material for biochemical studies.

Previously, luciferase-like enzymes were found in T. molitor mealworms and other beetle larvae (Viviani and Bechara, 1996). More recently, our group found and cloned one of these luciferase-like enzymes from the Malpighian tubules in Z. morio larvae (Coleoptera: Tenebrionidae). This luciferase-like enzyme is a Co-A ligase which displays weak luminescence activity in the presence of adenosine triphosphate (ATP) and firefly D-luciferin, a xenobiotic for this non-biouminescent organism (Viviani et al., 2009). Although the Malpighian tubules of this Coleoptera species are not bioluminescent, the presence of ATP-dependent luciferaselike enzymes is noteworthy, because the very distant dipteran relatives Arachnocampa spp display ATP-dependent bioluminescence, arising from lanterns consisting of specialized extensions of the Malpighian tubules (Gatenby, 1960; Viviani et al., 2002).

AMP-CoA ligases is a large family of enzymes found in all organisms, which activate carboxylic acid through ATP-dependent adenylation and usually followed by thioesterification to CoA for a wide variety of biological purposes, including fatty acid transport, pigment biosynthesis in plants, and aromatic compound detoxification, among others (Viviani, 2002). Thus, it would be interesting to decipher the biological role of AMP-CoA ligases with luciferase-like activity in the Malpighian tubules.

Thus, with the aim to better understand the molecular physiology of Malpighian tubules and the function of luciferase-like enzymes in these organs, we carried out the first transcriptional analysis using the previously constructed cDNA library from Malpighian tubules of the giant mealworm, Z. morio (Viviani et al., 2009).

\section{MATERIAL AND METHODS}

\section{cDNA library and excision}

A cDNA library was previously constructed in the $\lambda$ UNI-ZAP II vector (STRATAGENE, LaJolla, CA) using mRNA isolated from the Malpighian tubules of $Z$. morio (Viviani et al., 2009). The original library had a titer of $2 \times 10^{6}$ PFU. The $\lambda$ ZAP library was excised to yield a pBluescript plasmid library using Escherichia coli SOLR cells. Recombinant colonies were isolated on LB medium/agar plates by white/blue color selection in the presence of 10 $\mu \mathrm{g} / \mathrm{mL}$ 5-bromo-4-chloro-3-indolyl-beta-D-galactopyranoside and $100 \mu \mathrm{g} / \mathrm{mL}$ isopropyl betaD-thiogalactopyranoside).

\section{Extraction and analysis of plasmid DNA from recombinant colonies}

Plasmid DNA was extracted using the Wizard Plus SV DNA Purification System kit (PROMEGA), following the centrifugation protocol of the company. The analysis of the quality and size of the extracted plasmid DNA was performed by electrophoresis after digestion with EcoRI on a $1 \%(w / v)$ agarose gel in $1 \mathrm{X}$ TAE buffer (Tris-acetate-EDTA) at $100 \mathrm{~V}$ for $40 \mathrm{~min}$ and revealed by fluorescence with Blue Green (LGC, Brazil) under a UV transilluminator. 


\section{DNA sequencing}

Sequencing was carried out at Instituto de Química (Universidade de São Paulo, São Paulo) and Technology Departament of Faculdade de Ciências Agrárias e Veterinárias (Universidade Estadual Paulista Júlio de Mesquita Filho, Jaboticabal campus), according to the dideoxy (Sanger) method. The primers SK, KS and M13 were used at $10 \mu \mathrm{M}$. The sequences obtained had variable sizes between 200 and $600 \mathrm{bp}$.

\section{Bioinformatics}

The resulting electropherograms were read and edited with the BioEdit version 7.0.0 software. The 537 expression sequence tags (ESTs) obtained, E-value $\leq 5$, were compared with homologous sequences in the NCBI database through the BLAST algorithm (BLASTn and BLASTx; http://blast.ncbi.nlm.nih.gov/Blast) and SwissProt database (http://www.uniprot.org). The gene products were analyzed by homology-based GO annotation.

\section{RESULTS AND DISCUSSION}

We randomly isolated, partially sequenced and analyzed 537 clones obtaining a profile of the most representative expressed transcripts in Malpighian tubules of the mealworm $Z$. morio, associating them with their possible biological functions. The gene products analyzed by homology-based GO annotation could be divided into three categories: biological process (Figure 1), cellular component (Figure 2) and molecular function (Figure 3).

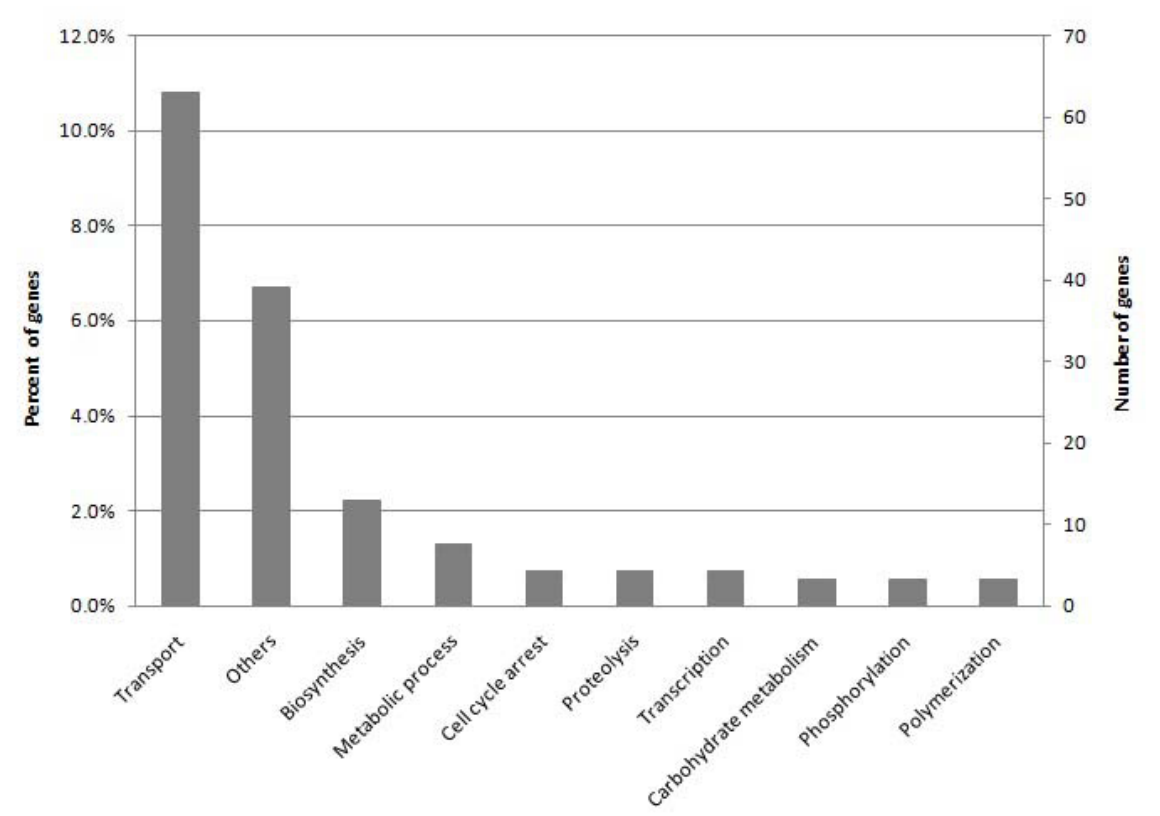

Figure 1. Genic products identified from Malpighian tubules of Zophobas morio larvae classified according to biological process. 


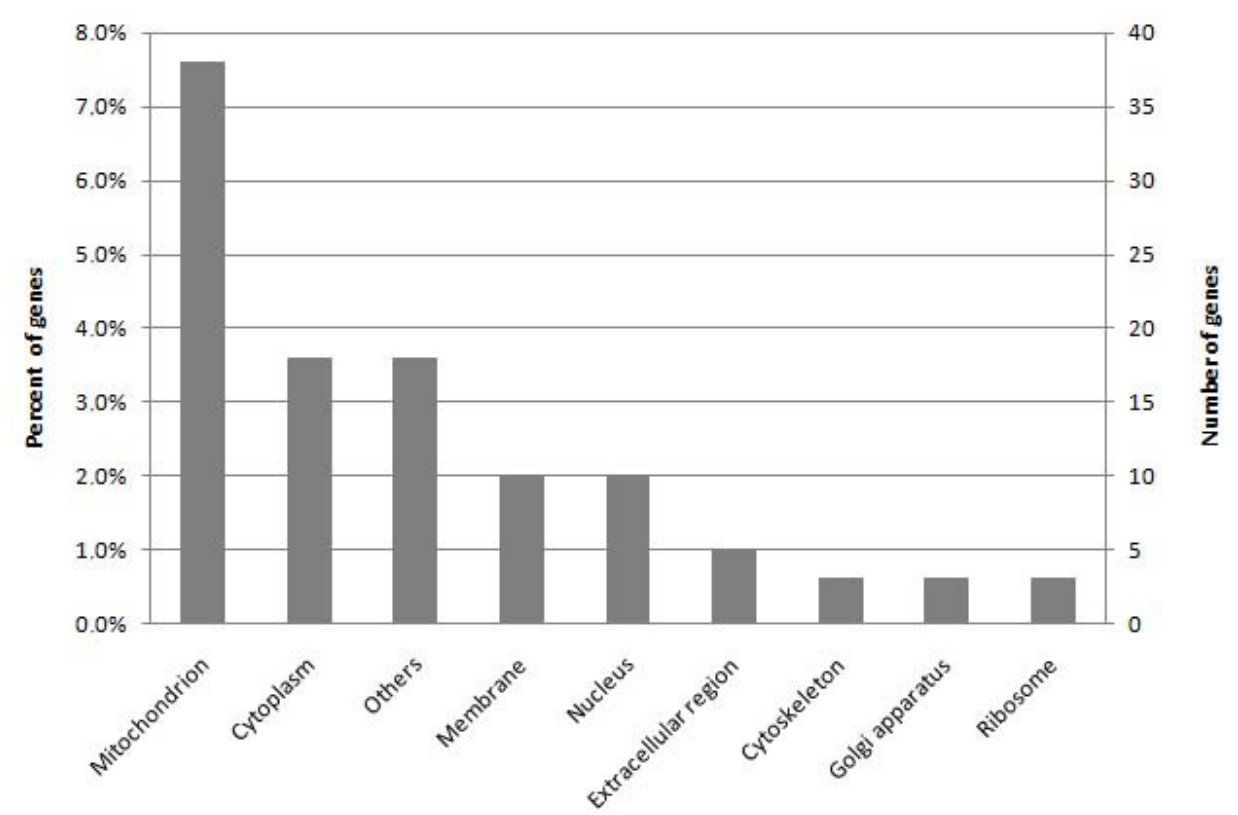

Figure 2. Genic products identified from Malpighian tubules of Zophobas morio larvae classified according to cellular component.

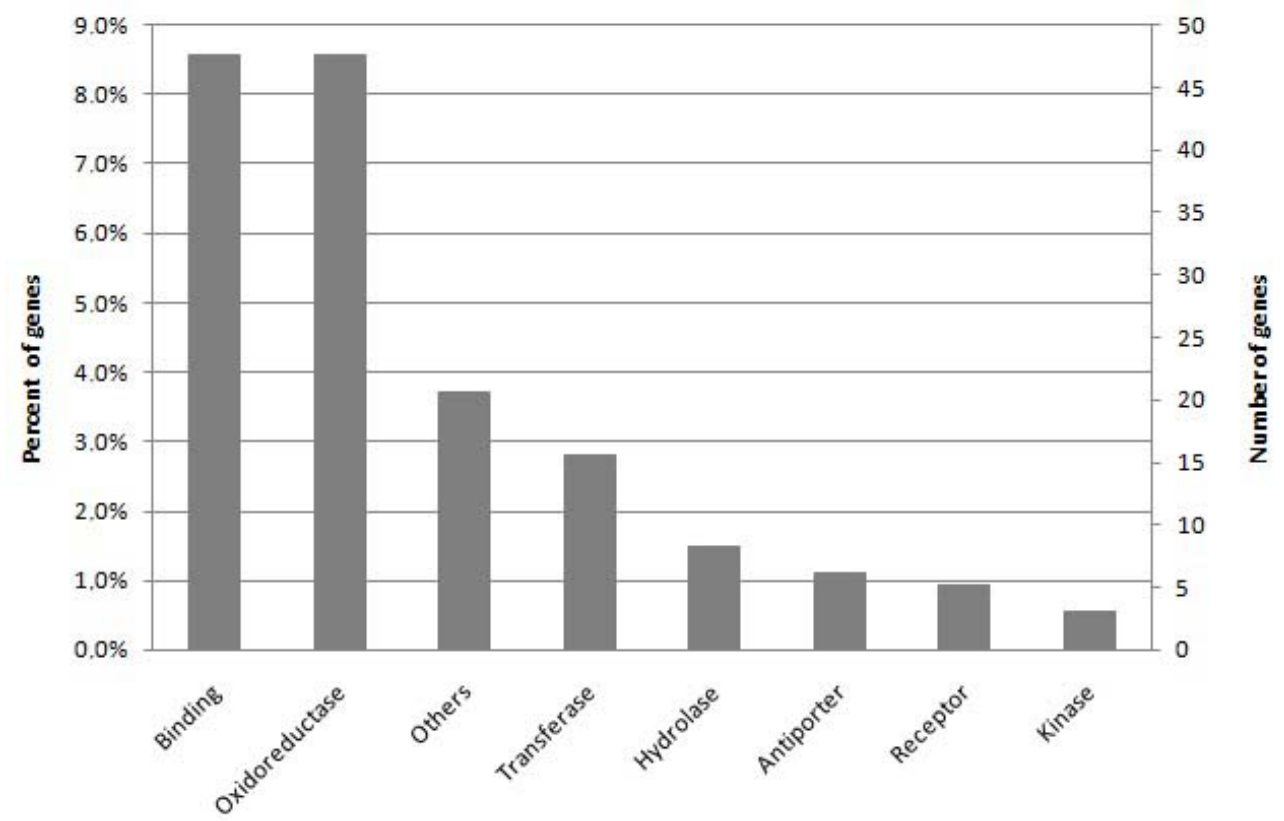

Figure 3. Genic products identified from Malpighian tubules of Zophobas morio larvae classified according to molecular function. 
The biological functions inferred of the main classes of gene products represented in this cDNA library were: oxidoreductases $(8.6 \%)$, transporters $(10.8 \%)$, antiporters $(1.1 \%)$, transferases $(2.8 \%)$, binding proteins $(8.6 \%)$, receptors $(1 \%)$, and hydrolases $(1.5 \%)$.

As expected, several sequences displayed high similarity to coded proteins of the genome of $T$. castaneum, which is a very closely related to Z. morio, with similar feeding requirements. Several genes of unknown function were found (16.6\%), which could be involved in important metabolic functions in Malpighian tubules. There was an abundance of transporter proteins and mitochondrial proteins involved in respiratory chain transport. We also found the following: genes similar to that of short-chain dehydrogenase, belonging to a large family of oxido-reductases involved in phase I reaction of xenobiotic detoxification (Oppermann and Maser, 2000; Kavanagh et al., 2008); cytochrome P450 genes which are involved in the detoxification of aromatic compounds and insecticide resistance; transcription factors; hydroxyacyl-CoA dehydrogenase-like enzymes, which may participate in fatty acid metabolism; and serine carboxypeptidase-like enzymes, which are involved in secondary compound metabolism and carry out substrate conversions, such as fatty acids and phenolic acids into glucose (Li and Steffens, 2000; Chahine and O'Donnell, 2010). There was the presence of some ESTs similar to genes coding for serine/threonine protein phosphatases (AJ606472.1) and protein kinases (XP 970757.2); these products are likely to be involved in the hormonal control of fluid secretion in the Malpighian tubules (Wang et al., 2004).

In comparison, the transcriptional analysis of D. melanogaster Malpighian tubules also showed high expression of organic and inorganic solute transporters, gene products related to xenobiotic metabolism, several transcription factors, gene products involved in signaling pathways and many novel genes, which could be related to other Malpighian tubule functions (Wang et al., 2004; Dow and Davies, 2006). Therefore, our analysis with Zophobas mealworm Malpighian tubule transcripts was in general consistent with the analysis of $D$. melanogaster.

\section{Mitochondrial products}

Our analysis showed high representation of mitochondrial gene products such as cytochromes $\mathrm{b}$ and $\mathrm{c}$ and cytochrome oxidases. We also found genes similar to those encoding ATP synthase subunits and nicotinamide adenine dinucleotide (NADH) dehydrogenase subunits. The high expression of mitochondrial gene products in this tissue is consistent with the high energy demand for active transport of several compounds from the hemolymph into the Malpighian tubule lumen, participating in the generation of primary urine and excretion (Chapman, 1998).

\section{Transporters and ATPases}

As in the case of D. melanogaster, the Malpighian tubules of $Z$. morio also display high representation of specific transporters for sugars, organic cations, anions, amino acids, and carboxylic acids (Wang et al., 2004). We found ESTs similar to genes encoding: ADP/ATP carrier proteins (XP 973257.1), which catalyze the transport of adenine nucleotides through internal and external mitochondrial membrane; sugar transporters (XP 972140.1); and sodium/potassium/calcium exchanger 1 (NP 064475.1). In this analysis, we also found ESTs similar to genes encoding the multidrug ABC transporter ATPase (YP 004969918.1) and plasma membrane $\mathrm{H}^{+}$-ATPase (CAL35828.1). 
Adenine nucleotide translocase, ANT, is located in the mitochondrial inner membrane and performs ADP/ATP exchange. ANT has a key role in the maintenance of cellular energy homeostasis, and in Drosophila it is associated with mechanical stress. This protein is encoded by the $\operatorname{Ses} B$ gene, and it has been shown that the presence of $\operatorname{Ses} B$ RNAi in the tubules impacts ATP production, reducing the mitochondrial calcium level and fluid transport rate and increasing $\mathrm{H}_{2} \mathrm{O}_{2}$ production. The use of SesB RNAi in the tubules of Drosophila shows that the absence ANT in the pupa is lethal and that there is an overexpression of this protein in larvae (Terhzaz et al., 2010).

ATPases are involved in many cellular functions, including acidification of cellular compartments, endocytosis and degradation of proteins and coupled transport of small molecules (Nishi and Forgac, 2002; Shinohara et al., 2010).

Malpighian tubule V-type $\mathrm{H}^{+}$ATPase (vacuolar-type proton pump) performs the transepithelial secretion of electrolytes and other compounds. The $\mathrm{H}^{+}$-pumping ATPase is located at the apical membrane of tubular cells, and it carries out the translocation of $\mathrm{H}^{+}$from the cytoplasm to the tubule lumen generating electrochemical potentials. These electrochemical potentials can drive the transport of $\mathrm{Na}^{+}, \mathrm{K}^{+}$and other solutes from the cytoplasm to the Malpighian tubule lumen (Beyenbach et al., 2010). In Drosophila, the disruption of V-ATPase may influence normal embryonic development, and in humans V-type $\mathrm{H}^{+}$ATPase mutations lead to renal tubular acidosis and sensory deafness (Zhong et al., 2013).

$\mathrm{ABC}$ transporters use ATP hydrolysis as a source of energy to transport drugs, lipids, sugars, ions, and amino acids across the cell membrane. $\mathrm{ABC}$ transporters have two nucleotidebinding domains and two transmembrane domains (Chang, 2003). In insects, these transporters participate in uric metabolism, development and possibly insecticide resistence. An analysis of $\mathrm{ABC}$ transporter expression in Bombyx mori revealed that the Malpighian tubule is enriched with these transporters, suggesting a possible important function in this tissue (Liu et al., 2011).

The presence of specific transporters in tubules is related to the need for the transport of a wide range of metabolic byproducts and xenobiotcs from the hemolymph to the Malpighian tubule lumen during the formation of primary urine and its excretion (Wang et al., 2004).

\section{Xenobiotic metabolism}

The xenobiotic metabolism can be divided into phase-I and phase-II detoxification pathways. Phase-I enzymes are mostly P450 monoxygenases (P450s), which introduce polar groups in their substrates through oxidation or hydrolysis. In the phase-II pathway, xenobiotics are conjugated with other compounds, i.e., sulfate, glutathione, amino acids, or CoA (Chahine and O'Donnell, 2010). Phase-I and -II enzymes usually make xenobiotics more water-soluble and easily excreted into urine, where they are involved in resistence to insecticides (Yang et al., 2007). After phase I and II, the products are eliminated through various transporters, such as members of the ATPbinding cassette superfamily of transporters (Chahine and O'Donnell, 2010).

In our analysis, we also found ESTs similar to genes encoding chaperones or heat shock proteins, which are evolutionarily conserved in the animal kingdom, from bacteria to humans. In bees, chaperones are expressed in Malpighian tubules and intestine, where their activation may aid in the resistance to xenobiotic compounds. This mechanism is still unknown and may involve regulated signaling cascades, which lead to survival mechanisms and programmed cell death when there is induction by environmental stress or toxic compounds (Silva-Zacarin et al., 2010). 


\section{P450 enzymes}

Cytochromes P450 enzymes are usually found in the endoplasmic reticulum of eukaryotes, participating in the metabolism of endogenous compounds and xenobiotics through NADPH-dependent oxidations. Cytochrome P450s are found in a large number of isoforms which display a wide range of substrates in different tissues and organisms (Scott, 1999). The cytochrome P450 families 1-4 play a role in phase-I xenobiotic detoxification in plants, invertebrates and vertebrates (Baldwin et al., 2009). We found sequences similar to genes coding for P450 such as isoform 1, CYP4Q2 and CYP4Q3. In insects, the CYP4 family is involved in fatty acid metabolism and juvenile hormone biosynthesis. The presence of $\mathrm{P} 450$ in insects has been also associated with higher resistance to insecticides and chemical compounds found in their food. The function of the CYP4Q2 family in animals can be divided into two main categories: synthesis of endogenous compounds and metabolism of endogenous or exogenous compounds from the diet or environment, such as insecticides or plant allelochemicals. In mammals, this family of enzymes is also involved in xenobiotic metabolism (Baldwin et al., 2009). In T. castaneum, the CYP4 family is probably involved with environmental responses. The high expansion of the CYP4 genes in this species could be involved in adaptation to detoxifying environmental chemicals found in the diet (Tribolium Genome Sequencing Consortium et al., 2008).

\section{AMP-CoA ligases}

The functional screening of ca. 3000 clones of Z. morio Malpighian tubule cDNA library yielded two copies for a luciferase-like enzyme, which was further characterized (Viviani et al., 2009). In this transcriptional survey of the same cDNA library, we did not find other ESTs similar to genes codings for luciferase-like enzymes. The limited number of ESTs analyzed in this study $(\sim 500)$ may explain the absence of further copies. The superfamily of AMP-CoA ligases includes bifunctional enzymes that catalyze I) the adenylation of carboxylic substrates at the expense of ATP and usually II) their thioesterification to CoA (Knights and Drogemuller, 2000; Viviani, 2002). This class of enzymes participate in various metabolic pathways, such as pigment biosynthesis in plants, fatty acid transport, production of acetate by acetate-CoA ligases, activation of fatty acids by acyl-CoA ligases, and even bioluminescence in beetles, among other functions (Karan et al., 2001; Viviani, 2002). They display highly conserved motifs for AMP binding. Some of these enzymes, especially mammalian long chain fatty-acyl-CoA synthetases found in liver and kidneys, may also be involved in the detoxification of xenobiotics via type II conjugation with amino acids (Knights and Drogemuller, 2000). The luciferase-like enzyme previously cloned from the Malpighian tubules of Z. morio larvae produces weak luminescence in the presence of ATP and firefly D-luciferin, a benzothiazolic compound that is a xenobiotic for the mealworm (Viviani et al., 2009). This weak luminescence activity with D-luciferin is the result of an oxidative side-reaction (Viviani et al., 2009). The presence of such enzyme in Malpighian tubules which display excretion and detoxification functions, the presence of targeting signals for the endoplasmic reticulum, which is an organelle also involved in drug detoxification metabolism, the participation of some AMP-ligases in phase-II detoxification reactions, and the specific oxidative reactivity for D-luciferin, which is a xenobiotic for this enzyme, provide compelling lines of evidences for a detoxification function for this enzyme in the Malpighian tubules. This luciferase-like enzyme 
may have a xenobiotic detoxification function in the Malpighian tubules, analogous to that of mammalian kidney fatty-acyl CoA synthetases.

\section{Concluding Remarks}

We carried out a transcriptional analysis of the most representative ESTs found in the Malpighian tubules of the mealworm Z. morio, providing the first transcriptional analysis of Malpighian tubules in a coleopteran species. The results confirm the reputation of Malpighian tubules as a very metabolically active and versatile organ, displaying a wide range of functions, especially organic compound transport and detoxification. Enzymes involved in phaseII reactions of detoxification were particularly abundant, especially oxidases. The interesting presence of AMP-CoA ligases with luciferase/oxygenase activity towards the xenobiotic firefly D-luciferin, suggests their potential involvement in new important excretive/detoxificative functions in Malpighian tubule physiology via adenylation/thioesterification and oxidation.

\section{ACKOWLEDGMENTS}

Research supported by Fundação de Amparo a Pesquisa do Estado de São Paulo (FAPESP, Brazil) and Conselho Nacional de Desenvolvimento Científico e Tecnológico (CNPq, Brazil). The authors thank Prof. Yoshihiro Ohmiya (AIST, Tsukuba) for revising and discussing the manuscript and Lucy Navarro (Instituto de Química, Universidade de São Paulo, São Paulo) and Agda Facincani (Technology Departament of Faculdade de Ciências Agrárias e Veterinárias, Universidade Estadual Paulista Júlio de Mesquita Filho, Jaboticabal campus) for DNA sequencing.

\section{REFERENCES}

Baldwin WS, Marko PB and Nelson DR (2009). The cytochrome P450 (CYP) gene superfamily in Daphnia pulex. BMC Genomics 10: 169.

Beyenbach KW, Skaer H and Dow JA (2010). The developmental, molecular, and transport biology of Malpighian tubules. Annu. Rev. Entomol. 55: 351-374.

Chahine S and O'Donnell MJ (2010). Effects of acute or chronic exposure to dietary organic anions on secretion of methotrexate and salicylate by Malpighian tubules of Drosophila melanogaster larvae. Arch. Insect Biochem. Physiol. 73: 128-147.

Chang G (2003). Multidrug resistance ABC transporters. FEBS Lett. 555: 102-105.

Chapman RF (1998). Excretion and Salt and Water Regulation. In: The Insect: Structure and Function. 4th edn. Cambridge University Press, London, 478-508.

Dow JA and Davies SA (2006). The Malpighian tubule: rapid insights from post-genomic biology. J. Insect Physiol. 52: 365-378.

Gaertner LS, Murray CL and Morris CE (1998). Transepithelial transport of nicotine and vinblastine in isolated Malpighian tubules of the tobacco hornworm (Manduca sexta) suggests a P-glycoprotein-like mechanism. J. Exp. Biol. 201: 2637-2645.

Gatenby JB (1960). The Australasian Mycetophilid Glowworms. Trans. Proc. Roy Soc. New Zeland. 88: 577-593.

Karan D, David JR and Capy P (2001). Molecular evolution of the AMP-forming Acetyl-CoA synthetase. Gene 265: 95-101.

Kavanagh KL, Jörnvall H, Persson B and Oppermann U (2008). Medium- and short-chain dehydrogenase/reductase gene and protein families: the SDR superfamily: functional and structural diversity within a family of metabolic and regulatory enzymes. Cell Mol. Life Sci. 65: 3895-3906.

Klowden JM (2007). Excretory Systems. In: Physiological System in Insects. 2nd edn. Academic Press, 403-431. 
Knights KM and Drogemuller CJ (2000). Xenobiotic-CoA ligases: kinetic and molecular characterization. Curr. Drug Metab. 1: 49-66.

Krueger RA, Broce AB, Hopkins TL and Kramer KJ (1988). Calcium transport from Malpighian tubules to puparial cuticle of Musca autumnalis. J. Comp. Physiol. 158: 413-419.

Li AX and Steffens JC (2000). An acyltransferase catalyzing the formation of diacylglucose is a serine carboxypeptidaselike protein. Proc. Natl. Acad. Sci. U. S. A. 97: 6902-6907.

Liu S, Zhou S, Tian L, Guo E, et al. (2011). Genome-wide identification and characterization of ATP-binding cassette transporters in the silkworm, Bombyx mori. BMC Genomics 12: 491.

Maddrell SH and Gardiner BO (1976). Excretion of alkaloids by malpighian tubules of insects. J. Exp. Biol. 64: 267-281.

Nappi AJ, Vass E, Frey F and Carton Y (2000). Nitric oxide involvement in Drosophila immunity. Nitric Oxide 4: 423 430.

Nishi T and Forgac M (2002). The vacuolar (H+)-ATPases--nature's most versatile proton pumps. Nat. Rev. Mol. Cell Biol. 3: 94-103.

Oppermann UC and Maser E (2000). Molecular and structural aspects of xenobiotic carbonyl metabolizing enzymes. Role of reductases and dehydrogenases in xenobiotic phase I reactions. Toxicology 144: 71-81.

Rafaeli-Bernstein A and Mordue W (1978). The transport of the cardiac glycoside ouabain by the Malpighian tubules of Zonocerus variegates. Physiol. Ent. 3: 59-63.

Ruiz-Sanchez E, Van Walderveen MC, Livingston A and O’Donnell MJ (2007). Transepithelial transport of salicylate by the Malpighian tubules of insects from different orders. J. Insect Physiol. 53: 1034-1045.

Scott JG (1999). Cytochromes P450 and insecticide resistance. Insect Biochem. Mol. Biol. 29: 757-777.

Shinohara Y, Hashimoto M, Kihira Y, Ohkura K, et al. (2010). Structural and functional properties of the C-terminal region of mitochondrial ADP/ATP carrier. Yakugaku Zasshi 130: 199-204.

Silva-Zacarin ECM, Ferreira RAC, Nocelli RCF, Roat TC, et al (2010). Structure and Function of the Intestine and Malpighian Tubules: From bee Biology to cell Marker Development for Toxicological Analysis. In: Stewart EM Social Insects: Structure, Function, and Behavior Nova Publishers, 121-142.

Terhzaz S, Cabrero P, Chintapalli VR, Davies SA, et al. (2010). Mislocalization of mitochondria and compromised renal function and oxidative stress resistance in Drosophila SesB mutants. Physiol. Genomics 41: 33-41.

Torrie LS, Radford JC, Southall TD, Kean L, et al. (2004). Resolution of the insect ouabain paradox. Proc. Natl. Acad. Sci. U. S. A. 101: 13689-13693.

Tribolium Genome Sequencing Consortium, Richards S, Gibbs RA, Weinstock GM, et al. (2008). The genome of the model beetle and pest Tribolium castaneum. Nature 452: 949-955.

Viviani VR (2002). The origin, diversity, and structure function relationships of insect luciferases. Cell Mol. Life Sci. 59: 1833-1850.

Viviani VR and Bechara EJH (1996). Larval Tenebrio molitor (Coleoptera: Tenebrionidae) fat body extracts catalyze firefly d-luciferin- and ATP-dependent chemiluminescence: a luciferase-like enzyme. Photochem. Photobiol. 63: 713-718.

Viviani VR, Hastings JW and Wilson T (2002). Two bioluminescent diptera: the North American Orfelia fultoni and the Australian Arachnocampa flava. Similar niche, different bioluminescence systems. Photochem. Photobiol. 75: 22-27.

Viviani VR, Prado RA, Arnoldi FC and Abdalla FC (2009). An ancestral luciferase in the Malpighi tubules of a nonbioluminescent beetle! Photochem. Photobiol. Sci. 8: 57-61.

Wang J, Kean L, Yang J, Allan AK, et al. (2004). Function-informed transcriptome analysis of Drosophila renal tubule. Genome Biol. 5: R69.

Wigglesworth VB (1972). The Principles of Insect Physiology. 7th edn. Chapman and Hall, London.

Yang J, McCart C, Woods DJ, Terhzaz S, et al. (2007). A Drosophila systems approach to xenobiotic metabolism. Physiol. Genomics 30: 223-231.

Zhong XW, Zou Y, Liu SP, Yi QY, et al. (2013). Proteomic-based insight into Malpighian tubules of silkworm Bombyx mori. PLoS One 8: e75731. 